\title{
WEIGHTED NORM INEQUALITIES FOR HILBERT TRANSFORMS AND CONJUGATE FUNCTIONS OF EVEN AND ODD FUNCTIONS ${ }^{1}$
}

\author{
KENNETH F. ANDERSEN
}

\begin{abstract}
It is well known that the Hilbert tranformation and the conjugate function operator restricted to even (odd) functions define bounded linear operators on weighted $L^{p}$ spaces under more general conditions than is the case for the unrestricted operators. In analogy with recent results of Hunt, Muckenhoupt and Wheeden for the Hilbert transform and the conjugate function operator, we obtain necessary and sufficient conditions in order that these restricted operators should satisfy weighted weak-type inequalities and hence also necessary and sufficient conditions in order that these operators should be bounded on weighted $L^{p}$ spaces for $1<p<\infty$.
\end{abstract}

1. Introduction. Let $H$ denote the Hilbert transformation given by the Cauchy principal value integral

$$
(H f)(x)=\frac{1}{\pi} \int_{-\infty}^{\infty} \frac{f(t)}{t-x} d t, \quad x \in(-\infty, \infty) .
$$

If $f$ is odd, then $H f$ is even and given by $(H f)(x)=\left(H_{\mathrm{o}} f\right)(|x|)$ where

$$
\left(H_{\mathrm{o}} f\right)(x)=\frac{2}{\pi} \int_{0}^{\infty} \frac{t f(t)}{t^{2}-x^{2}} d t, \quad(x>0),
$$

and if $f$ is even then $H f$ is odd and given for $x>0$ by $(H f)(x)=\left(H_{\mathrm{e}} f\right)(x)$ where

$$
\left(H_{\mathrm{e}} f\right)(x)=\frac{2}{\pi} \int_{0}^{\infty} \frac{x f(t)}{t^{2}-x^{2}} d t .
$$

If $W$ is a nonnegative measurable function and $1 \leqslant p<\infty$, norm inequalities of the form

$$
\int_{\Omega}|(T f)(x)|^{p} W(x) d x \leqslant C_{p} \int_{\Omega}|f(x)|^{p} W(x) d x
$$

where $C_{p}$ is a constant depending on $p$ and $W$ but independent of $f$, $\Omega=(-\infty, \infty)$ if $T=H$, and $\Omega=(0, \infty)$ if $T=H_{\mathrm{o}}$ or $H_{\mathrm{e}}$ have been studied by many authors, as have the weak type inequalities

$$
\int_{\{x \in \Omega:|(T f)(x)|>y\}} W(x) d x \leqslant C_{p} y^{-p} \int_{\Omega}|f(x)|^{p} W(x) d x, \quad(y>0) .
$$

Received by the editors October 28, 1974.

AMS (MOS) subject classifications (1970). Primary 44A15, 42A40; Secondary 26A86.

${ }^{1}$ Research supported in part by NRC Grant \#A-8185. 
For the special case $W(x) \equiv 1$, it is well known that (1.1) holds if and only if $1<p<\infty$, while (1.2) holds for all $p, 1 \leqslant p<\infty$. For $W$ of the form $W(x)=|x|^{\alpha-1}$, and $1<p<\infty$, Babenko [1] states that (1.1) holds for $T=H$ if $0<\alpha<p$, whereas a result of Hardy and Littlewood [5] states that (1.1) holds for $T=H_{\mathrm{e}}$, if $\alpha$ satisfies $-p<\alpha<p$, and recently Rooney [8] has shown that (1.1) is true for $T=H_{\mathrm{o}}$ if $\alpha$ satisfies $0<\alpha<2 p$.

The problem of characterizing those $W$ satisfying (1.1) for $T=H$ has attracted many authors, but only recently has the complete solution been obtained. Hunt, Muckenhoupt and Wheeden [6] have shown that $W$ satisfies (1.1) for $1<p<\infty$ if and only if:

there is a constant $K$ such that for every $[a, b] \subset(-\infty, \infty)$,

$$
\left(\int_{a}^{b} W(x) d x\right)\left(\int_{a}^{b} W(x)^{-1 /(p-1)} d x\right)^{p-1} \leqslant K|b-a|^{p} .
$$

They also proved that (1.2) holds for $T=H, 1 \leqslant p<\infty$, if and only if (1.3) holds. Condition (1.3) is referred to as the $A_{p}$ condition and it is to be understood that $0 \cdot \infty$ is taken to be 0 while for $p=1$, the second factor on the left of (1.3) is taken to be ess $\sup _{x \in[a, b]} W(x)^{-1}$.

In $\$ 2$ of this paper we obtain the corresponding characterization of those $W$ satisfying (1.1) or (1.2) when $T$ is either $H_{\mathrm{o}}$ or $H_{\mathrm{e}}$.

The problems corresponding to (1.1) and (1.2) when $T$ is the periodic analogue of $H$, the conjugate operator $C$, given by

$$
(C f)(\theta)=\frac{1}{2 \pi} \int_{-\pi}^{\pi} \cot \left(\frac{\phi-\theta}{2}\right) f(\phi) d \phi
$$

have also been widely studied with the complete solution again being given in [6]. Here also, if $f$ is odd (even), $C f$ is even (odd) and is given by $C f$ $=C_{\mathrm{o}} f\left(=C_{\mathrm{e}} f\right)$ where

$$
\begin{array}{ll}
\left(C_{\mathrm{o}} f\right)(\theta) & =\frac{1}{\pi} \int_{0}^{\pi} \frac{\sin \phi}{\cos \phi-\cos \theta} f(\phi) d \phi ; \\
\left(C_{\mathrm{e}} f\right)(\theta) & =\frac{1}{\pi} \int_{0}^{\pi} \frac{\sin \theta}{\cos \phi-\cos \theta} f(\phi) d \phi,
\end{array}
$$

and norm inequalities for these operators have also been given by Hardy and Littlewood [5], K. K. Chen [2], T. M. Flett [4], and Y.-M. Chen [3]. In \$3 we obtain a characterization of those $W$ which satisfy (1.1) for $1<p<\infty$ and $T=C_{\mathrm{o}}=C_{\mathrm{e}}(\Omega=(0, \pi))$ and also of those which satisfy $(1.2)$ for $1 \leqslant p$ $<\infty$ with $T=C_{\mathrm{o}}\left(=C_{\mathrm{e}}\right)$.

We adopt the usual practice that $A, B, C, K$ denote absolute constants, not necessarily the same at each occurrence.

2. Results for $H_{\mathrm{o}}$ and $H_{\mathrm{e}}$. We shall prove the following theorems:

THEOREM 1. Let $W(x)$ be nonnegative and measurable on $(0, \infty)$. If $1<p$ $<\infty$, then (a), (b) and (c) are equivalent; if $p=1$, then (a) and (b) are equivalent where: 
(a) There exists a constant $A_{p}$ such that for every $[a, b] \subset(0, \infty)$,

$$
\left(\int_{a}^{b} W(x) d x\right)\left(\int_{a}^{b} x^{p /(p-1)} W(x)^{-1 /(p-1)}\right)^{p-1} \leqslant A_{p}\left(\frac{b^{2}-a^{2}}{2}\right)^{p} .
$$

(b) There is a constant $B_{p}$ such that for all $y>0$,

$$
\int_{\left\{x>0:\left|\left(H_{0} f\right)(x)\right|>y\right\}} W(x) d x \leqslant B_{p} y^{-p} \int_{0}^{\infty}|f(x)|^{p} W(x) d x .
$$

(c) There is a constant $C_{p}$ such that

$$
\int_{0}^{\infty}\left|\left(H_{\mathrm{o}} f\right)(x)\right|^{p} W(x) d x \leqslant C_{p} \int_{0}^{\infty}|f(x)|^{p} W(x) d x .
$$

THEOREM 2. Let $W(x)$ be nonnegative and measurable on $(0, \infty)$. If $1<p$ $<\infty$, then (a), (b) and (c) are equivalent; if $p=1$ then (b) implies (a) where:

(a) There exists a constant $A_{p}$ such that for every $[a, b] \subset(0, \infty)$,

$$
\left(\int_{a}^{b} x^{p} W(x) d x\right)\left(\int_{a}^{b} W(x)^{-1 /(p-1)} d x\right)^{p-1} \leqslant A_{p}\left(\frac{b^{2}-a^{2}}{2}\right)^{p} .
$$

(b) There exists a constant $B_{p}$ such that for all $y>0$,

$$
\int_{\left\{x>0:\left|\left(H_{\mathrm{e}} f\right)(x)\right|>y\right\}} W(x) d x \leqslant B_{p} y^{-p} \int_{0}^{\infty}|f(x)|^{p} W(x) d x .
$$

(c) There exists a constant $C_{p}$ such that

$$
\int_{0}^{\infty}\left|\left(H_{\mathrm{e}} f\right)(x)\right|^{p} W(x) d x \leqslant C_{p} \int_{0}^{\infty}|f(x)|^{p} W(x) d x .
$$

For convenience we shall say that a $W$ satisfying (a) of Theorem 1 satisfies the $A_{p}^{\mathrm{o}}$ condition; a $W$ satisfying (a) of Theorem 2 satisfies the $A_{p}^{\mathrm{e}}$ condition. Further, when $p=1$, the second factor on the left in (a) of Theorem 1 is of course understood to be ess $\sup _{x \in[a, b]} x W(x)^{-1}$. Clearly $W(x)$ satisfies $A_{p}^{\mathrm{o}}$ if and only if $x^{-p} W(x)$ satisfies $A_{p}^{\mathrm{e}}$.

Note first that the $A_{p}$ condition (restricted to intervals $[a, b] \subset(0, \infty)$ ) implies both the $A_{p}^{\mathrm{e}}$ and $A_{p}^{\mathrm{o}}$ conditions; conversely if $W$ satisfies both $A_{p}^{\mathrm{e}}$ and $A_{p}^{\mathrm{o}}$, Hölder's inequality shows that

$$
\begin{gathered}
\left(\int_{a}^{b} x^{p} W(x) d x\right)\left(\int_{a}^{b} x^{p /(p-1)} W(x)^{-1 /(p-1)} d x\right)^{p-1} \\
\geqslant\left(\int_{a}^{b} x^{2} d x\right)^{p}=\left(\frac{b^{3}-a^{3}}{3}\right)^{p}
\end{gathered}
$$

and hence $W$ satisfies the $A_{p}$ condition on intervals $[a, b] \subset(0, \infty)$. Thus Theorems 1 and 2 are consistent with the result of Hunt, Muckenhoupt and Wheeden for $H$.

It may also be of interest to note here that if $1<p<\infty$, then Hölder's inequality shows that a $W$ satisfying $A_{p}^{\circ}$ satisfies $A_{2 p}$ on intervals $I \subset(0, \infty)$. 
The proof of Theorem 1 requires

LEMMA 1. Let $W$ be a nonnegative measurable function defined on $(0, \infty)$ and put $w(x)=W(\sqrt{ }|x|) / 2 \sqrt{ }|x|$ for $x \neq 0$. Then $W$ satisfies condition $A_{p}^{\circ}$ if and only if $w$ satisfies condition $A_{p}$. Further, if $W$ satisfies $A_{p}^{\circ}$ for some $p, 1<p<\infty$, then $W$ satisfies $A_{q}^{\mathrm{O}}$ for all $q>p-\epsilon$ for sufficiently small $\epsilon>0$.

Proof of Lemma 1. We give the proof for $1<p<\infty$, the case $p=1$ being entirely similar. Let $[a, b] \subset(0, \infty)$ or $[a, b] \subset(-\infty, 0)$. Then

$$
\begin{gathered}
\left(\int_{a}^{b} w(x) d x\right)\left(\int_{a}^{b} w(x)^{-1 /(p-1)} d x\right)^{p-1} \\
=\left(\int_{|a|}^{|b|} W(\sqrt{ } x) \frac{d x}{2 \sqrt{ } x}\right)\left(\int_{|a|}^{|b|}(2 \sqrt{ } x)^{p /(p-1)} W(\sqrt{ } x)^{-1 /(p-1)} \frac{d x}{2 \sqrt{ } x}\right)^{p-1} \\
=2^{p}\left(\int_{\sqrt{ }|a|}^{\sqrt{ }|b|} W(x) d x\right)\left(\int_{\sqrt{ }|a|}^{\sqrt{ }|b|} x^{p /(p-1)} W(x)^{-1 /(p-1)} d x\right)^{p-1}
\end{gathered}
$$

and therefore if $W$ satisfies the $A_{p}^{\circ}$ condition with constant $K$, the right side of (2.1) is bounded by $K|b-a|^{p}$, while for intervals $[a, b]$ with $a<0<b$ we have

$$
\begin{aligned}
& \left(\int_{a}^{b} w(x) d x\right)\left(\int_{a}^{b} w(x)^{-1 /(p-1)} d x\right)^{p-1} \\
& \quad \leqslant\left(2 \int_{0}^{c} w(x) d x\right)\left(2 \int_{0}^{c} w(x)^{-1 /(p-1)} d x\right)^{p-1}
\end{aligned}
$$

where $[-c, c]$ is the interval containing $[a, b]$ with at least one endpoint in common, hence the right side of $(2.2)$ is bounded by $K 2^{p} c^{p} \leqslant 2^{p} K|b-a|^{p}$ and $w$ satisfies the $A_{p}$ condition with constant $2^{p} K$. The converse follows immediately from (2.1).

Finally, if $w$ satisfies $A_{p}$ for some $p>1$, by [7, Lemma 5], $w$ satisfies $A_{p-\epsilon}$ for sufficiently small $\epsilon>0$ and, hence, by what we have just proved, if $W$ satisfies $A_{p}^{\circ}$ for some $p>1$, then $W$ satisfies $A_{p-\epsilon}^{\circ}$ for sufficiently small $\epsilon>0$, and Hölder's inequality shows that $W$ satisfies $A_{q}^{\mathrm{o}}, q>p$, if $W$ satisfies $A_{p}^{\mathrm{o}}$.

Proof of TheOREM 1. The main part of the proof consists in showing the equivalence of (a) and (b) for $1 \leqslant p<\infty$, for if this has been proved and if (b) holds for some $p_{\mathrm{o}}, 1<p_{\mathrm{o}}<\infty$, Lemma 1 shows that (b) must also hold for $p \geqslant p_{\mathrm{o}}-\epsilon$, sufficiently small $\epsilon>0$, and hence the Marcinkiewicz interpolation theorem [9, Volume II, p. 112] shows that (c) holds for $p=p_{\mathrm{o}}$, and since (c) always implies (b), the proof will be complete.

We prove first that (a) implies (b). Let $W$ satisfy (a), $W$ and $w$ be related as in Lemma 1 and suppose $f$ is given for which the right side of (b) is finite. If we write $g(x)=f(\sqrt{ } x)$ for $x>0$ and $g(x)=0$ otherwise, then

$$
\int_{-\infty}^{\infty}|g(x)|^{p} w(x) d x=\int_{0}^{\infty}|f(x)|^{p} W(x) d x<\infty .
$$

By Lemma 1,w satisfies $A_{p}$ and hence [6, Theorem 9] shows that $H g$ exists and 
for all $y>0$,

$$
\int_{\{x:|(H g)(x)|>y\}} w(x) d x \leqslant C y^{-p} \int_{0}^{\infty}|f(x)|^{p} W(x) d x .
$$

Now, if $x>0$,

$$
(H g)(x)=\frac{1}{\pi} \int_{0}^{\infty} \frac{f(\sqrt{ } t)}{t-x} d t=\frac{1}{\pi} \int_{0}^{\infty} \frac{2 t f(t)}{t^{2}-x} d t=\left(H_{\mathrm{o}} f\right)(\sqrt{ } x),
$$

and hence

$$
\begin{aligned}
\int_{\left\{x>0:\left|\left(H_{0} f\right)(x)\right|>y\right\}} W(x) d x & =\int_{\left\{x>0:\left|\left(H_{0} f\right)(\sqrt{ } x)\right|>y\right\}} w(x) d x \\
& \leqslant \int_{\left\{x:\left|\left(H_{g}\right)(x)\right|>y\right\}} w(x) d x,
\end{aligned}
$$

so it follows from (2.3) that (b) holds for some $B_{p}$.

Conversely, suppose (b) holds, and let $I=[a, b] \subset(0, \infty)$ and $J=[b, 2 b$ $-a]$.

Consider first the case that $1<p<\infty$. Let $f(x)=x^{1 /(p-1)} W(x)^{-1 /(p-1)}$ if $x \in I$ and $f(x)=0$ otherwise, and put

$$
A=\int_{0}^{\infty}|f(x)|^{p} W(x) d x=\int_{I} x^{p /(p-1)} W(x)^{-1 /(p-1)} d x .
$$

If $A=0$, by convention the left side of (a) is taken to be zero, so (a) holds in this case. If $A=\infty$, there exists $g \in L^{P}(I)$ such that $\int_{I} g(x) x W(x)^{-1 / p} d x$ $=\infty$ and if $h(x)=g(x) W(x)^{-1 / p}$, then $\left(H_{\mathrm{o}} h\right)(x)=\infty$ for $x \in J$ so that (b) implies

$$
\int_{J} W(x) d x \leqslant B_{p} y^{-p} \int_{I}|h(x)|^{p} W(x) d x=B_{p} y^{-p} \int_{I}|g(x)|^{p} d x
$$

for all $y>0$, and hence $\int_{J} W(x) d x=0$. But then $W(x)=0$ on $J$, so $\int_{J} x^{p /(p-1)} W(x)^{-1 /(p-1)} d x=\infty$, and now reversing the roles of $I$ and $J$ we obtain $\int_{I} W(x) d x=0$, so again the left side of (a) is zero and, therefore, (a) holds in this case also. Hence, we assume that $0<A<\infty$. If $x \in J$ we have

$$
\left|\left(H_{\mathrm{o}} f\right)(x)\right|=\frac{2}{\pi} \int_{I} \frac{t^{p /(p-1)} W(t)^{-1 /(p-1)}}{|(t-x)(t+x)|} d t \geqslant[\pi(3 b-a)(b-a)]^{-1} A,
$$

so (b) implies that

$$
\begin{aligned}
\int_{J} W(x) d x & \leqslant B_{p}[\pi(3 b-a)(b-a)]^{p} A^{-p} \int_{I}|f(x)|^{p} W(x) d x \\
& =B_{p}[\pi(3 b-a)(b-a)]^{p} A^{1-p} .
\end{aligned}
$$

Now put $g(x)=W(x)^{-1 /(p-1)}$ for $x \in J$ and $g(x)=0$ otherwise, and write

$$
B=\int_{J}|g(x)|^{p} W(x) d x=\int_{J} W(x)^{-1 /(p-1)} d x .
$$

If $B=0$, then $W(x)=\infty$ on $J$ which contradicts (2.4), and an argument 
similar to that given above for $A$, shows that $B<\infty$ unless $W(x)=0$ on $I$, i.e. unless $A=\infty$, hence we have $0<B<\infty$. For $x \in I$ we have

$$
\left|\left(H_{\mathrm{o}} g\right)(x)\right|=\frac{2}{\pi} \int_{J} \frac{t W(t)^{-1 /(p-1)}}{|(t-x)(t+x)|} d t \geqslant b[\pi(3 b-a)(b-a)]^{-1} B,
$$

so again by (b) we have

$$
\begin{aligned}
\int_{I} W(x) d x & \leqslant B_{p}[\pi(3 b-a)(b-a)]^{p} b^{-p} \int_{J}|g(x)|^{p} W(x) d x \\
& =B_{p}[\pi(3 b-a)(b-a)]^{p} b^{-p} B^{1-p} .
\end{aligned}
$$

Now if (2.4) and (2.5) are multiplied together and the resulting inequality multiplied by $(A B)^{p-1}$, then noting that by Hölder's inequality,

$$
\left(\int_{J} W(x) d x\right)\left(\int_{J} W(x)^{-1 /(p-1)} d x\right)^{p-1} \geqslant|b-a|^{p}
$$

we obtain

$$
\begin{gathered}
|b-a|^{p}\left(\int_{I} W(x) d x\right)\left(\int_{I} x^{p /(p-1)} W(x)^{-1 /(p-1)} d x\right)^{p-1} \\
\leqslant C|(3 b-a)(b-a)|^{2 p} b^{-p}
\end{gathered}
$$

which is easily seen to be equivalent to (a).

Finally we consider the case $p=1$. If ess $\inf _{x \in I} x^{-1} W(x)=\infty$, then (a) holds by convention, otherwise, let $\epsilon>0$ and choose $E \subset I, E$ of positive measure $|E|$, and $x^{-1} W(x) \leqslant \epsilon+\operatorname{ess}_{i \in I} t^{-1} W(t)$ for all $x \in E$. Let $f(t)=t^{-1}$ on $E, f(t)=0$ otherwise. Then for $x \in J$

$$
\left|\left(H_{\mathrm{o}} f\right)(x)\right|=\frac{2}{\pi} \int_{E} \frac{d t}{\left|t^{2}-x^{2}\right|} \geqslant[\pi(3 b-a)(b-a)]^{-1}|E|,
$$

and by (b),

$$
\begin{aligned}
\int_{J} W(x) d x & \leqslant B_{1}[\pi(3 b-a)(b-a)]|E|^{-1} \int_{E} t^{-1} W(t) d t \\
& \leqslant B_{1}[\pi(3 b-a)(b-a)]\left(\epsilon+\underset{x \in I}{\left.\operatorname{ess} \inf ^{-1} W(x)\right),}\right.
\end{aligned}
$$

and hence

$$
\int_{J} W(x) d x \leqslant B_{1}[\pi(3 b-a)(b-a)] \underset{x \in I}{\operatorname{ess} \inf } x^{-1} W(x) .
$$

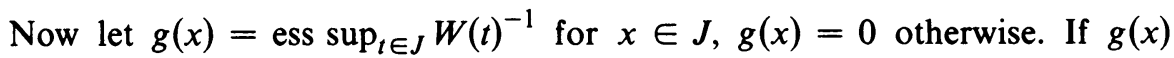
$=0$, then $W(t)=\infty$ a.e. on $J$, contradicting (2.6), while if $g(x)=\infty$, then ess $\inf _{x \in J} W(x)=0$, and an argument similar to that which leads to (2.6) shows that $\int_{I} W(x) d x=0$, so (a) holds in this case, and we may assume that $0<g(x)<\infty, x \in J$. Then if $\epsilon>0$, there exists $E \subset J,|E|>0$ such that 
$W(x) \leqslant \epsilon+\operatorname{ess} \inf _{t \in J} W(t)$ for $x \in J$, and if $h(x)=1$ for $x \in E, h(x)=0$, otherwise we have $\left|\left(H_{\mathrm{o}} h\right)(x)\right| \geqslant b[\pi(3 b-a)(b-a)]^{-1}|E|$ for $x \in I$, so (b) implies

$$
\begin{aligned}
\int_{I} W(x) d x & \leqslant B_{1}[\pi(3 b-a)(b-a)] b^{-1}|E|^{-1} \int_{E} W(x) d x \\
& =B_{1}[\pi(3 b-a)(b-a)] b^{-1}(\epsilon+\underset{t \in J}{\operatorname{essinf}} W(t))
\end{aligned}
$$

so that

$$
\int_{I} W(x) d x \leqslant B_{1}[\pi(3 b-a)(b-a)] b^{-1} \underset{t \in J}{\text { ess inf }} W(t) .
$$

Finally, if (2.6) and (2.7) are multiplied together and the resulting inequality multiplied by (ess $\left.\sup _{x \in I} x W(x)^{-1}\right)\left(\right.$ ess $\left.\sup _{x \in J} W(x)^{-1}\right)$, then, noting that

$$
\left(\underset{x \in J}{\operatorname{ess} \sup _{x}} W(x)^{-1}\right)\left(\int_{J} W(x) d x\right) \geqslant|J|=|b-a|,
$$

we obtain

$$
(b-a)\left(\int_{I} W(x) d x\right) \underset{x \in I}{\operatorname{ess} \sup } x W(x)^{-1} \leqslant C(3 b-a)^{2}(b-a)^{2} b^{-1},
$$

which clearly implies (a) and the proof is complete.

Proof of Theorem 2. The proof that (b) implies (a) for $1 \leqslant p<\infty$ is similar to that of the corresponding statement in Theorem 1 and is therefore omitted. Now let $1<p<\infty$. As observed above, $W(x)$ satisfies $A_{p}^{\mathrm{o}}$ if and only if $x^{-p} W(x)$ satisfies $A_{p}^{\mathrm{e}}$, and since $H_{\mathrm{o}}$ and $H_{\mathrm{e}}$ are related by

$$
\left(H_{\mathrm{e}} g\right)(x)=x\left(H_{\mathrm{o}} f\right)(x) \quad \text { where } g(t)=t f(t),
$$

it follows immediately from Theorem 1 that (a) and (c) are equivalent. But then if (b) is satisfied, (a) holds, so (c) holds and since (c) always implies (b), the proof is complete.

REMARK. Although our methods do not show it, we conjecture that in Theorem 2, (a) implies (b) even in the case $p=1$.

3. Results for $C_{\mathrm{o}}, C_{\mathrm{e}}$. Here we state the analogues of Theorems 1 and 2 for the periodic case, and indicate briefly how they may be derived.

THEOREM 3. Let $w(\theta)$ be nonnegative and measurable on $(0, \pi)$. If $1<p$ $<\infty$, (a), (b) and (c) are equivalent; if $p=1$, (a) and (b) are equivalent where:

(a) There is a constant $A_{p}$ such that for every $(a, b) \subset(0, \pi)$,

$$
\begin{gathered}
\left(\int_{a}^{b} w(\theta) d \theta\right)\left(\int_{a}^{b} \sin ^{p /(p-1)} \theta w(\theta)^{-1 /(p-1)} d \theta\right)^{p-1} \\
\leqslant A_{p} \sin ^{p}\left(\frac{b+a}{2}\right) \sin ^{p}\left(\frac{b-a}{2}\right) .
\end{gathered}
$$

(b) There is a constant $B_{p}$ such that for all $y>0$, 


$$
\int_{\left\{\theta:\left|\left(C_{\mathrm{o}} f\right)(\theta)\right|>y\right\}} w(\theta) d \theta \leqslant B_{p} y^{-p} \int_{0}^{\pi}|f(\phi)|^{p} w(\phi) d \phi .
$$

(c) There is a constant $C_{p}$ such that

$$
\int_{0}^{\pi}\left|\left(C_{\mathrm{o}} f\right)(\theta)\right|^{p} w(\theta) d \theta \leqslant C_{p} \int_{0}^{\pi}|f(\phi)|^{p} w(\phi) d \phi .
$$

THEOREM 4. Let $w(\theta)$ be nonnegative and measurable on $(0, \pi)$. If $1<p$ $<\infty$, then (a), (b) and (c) are equivalent; if $p=1$, (b) implies (a) where:

(a) There is a constant $A_{p}$ such that for every $(a, b) \subset(0, \pi)$,

$$
\begin{gathered}
\left(\int_{a}^{b} \sin ^{p} \theta w(\theta) d \theta\right)\left(\int_{a}^{b} w(\theta)^{-1 /(p-1)} d \theta\right)^{p-1} \\
\leqslant A_{p} \sin ^{p}\left(\frac{b+a}{2}\right) \sin ^{p}\left(\frac{b-a}{2}\right) .
\end{gathered}
$$

(b) There is a constant $B_{p}$ such that for all $y>0$,

$$
\int_{\left\{\theta:\left|\left(C_{\mathrm{e}} f\right)(\theta)\right|>y\right\}} w(\theta) d \theta \leqslant B_{p} y^{-p} \int_{0}^{\pi}|f(\phi)|^{p} w(\phi) d \phi .
$$

(c) There is a constant $C_{p}$ such that

$$
\int_{0}^{\pi}\left|\left(C_{\mathrm{e}} f\right)(\theta)\right|^{p} w(\theta) d \theta \leqslant C_{p} \int_{0}^{\pi}|f(\phi)|^{p} w(\phi) d \phi .
$$

The proof in Theorem 3 that (a) $\Rightarrow$ (b) may be patterned after that of Theorem 1 with Lemma 1 replaced by

LeMMA 2. Let $w(\theta)$ be nonnegative and measurable on $(0, \pi)$. Let $W(x)$ be the function on $(-\infty, \infty)$ which is of period 2 , even on $(-1,1)$ and given by

$$
W(x)=w\left(2 \sin ^{-1} \sqrt{ } x\right) /(x(1-x))^{1 / 2}
$$

when $x \in(0,1)$. If $1 \leqslant p<\infty, W$ satisfies the $A_{p}$ condition if and only if $w$ satisfies condition (a) of Theorem 3 , in particular if $w$ satisfies that condition for some $p>1$, then it also satisfies the condition for all $q, q>p-\epsilon$, sufficiently small $\epsilon>0$.

Proof of Lemma 2. For intervals $(a, b)$ containing at most one integer, the outline of the proof of Lemma 1 may be followed to obtain a constant $K$ for which the desired inequality for $W$ holds, then the periodicity of $W$ shows that the constant $3^{p} K$ will suffice for an arbitrary interval.

Proof of Theorem 3. The proof of Theorem 1 may be imitated with obvious modifications, except that in the proof that $(\mathrm{b}) \Rightarrow$ (a), the interval $J$ corresponding to $I$ is now chosen so that $J$ and $I$ have exactly one endpoint in common, have the same length, and are both contained in $(0, \pi)$. This is possible since, by periodicity, it is only necessary to consider intervals $I$ of length less that $\pi / 4$.

Proof of Theorem 4. Since $\left(C_{\mathrm{e}} g\right)(\theta)=\sin \theta\left(C_{\mathrm{o}} f\right)(\theta)$, where $g(\phi)$ $=\sin \phi f(\phi)$, and $w$ satisfies condition (a) of Theorem 4 if and only if 
$\sin ^{p} \theta w(\theta)$ satisfies (a) of Theorem 3 , Theorem 4 follows from Theorem 3 in the same way that Theorem 2 followed from Theorem 1.

REMARK. We conjecture that (a) $\Rightarrow(b)$ in Theorem 4 for the case $p=1$ also, although our methods do not show this.

\section{REFERENCES}

1. K. I. Babenko, On conjugate functions, Dokl. Akad. Nauk SSSR 62 (1948), 157-160. (Russian) MR 10, 249.

2. K.-K. Chen, On the absolute Cesàro summability of negative order for a Fourier series at a given point, Amer. J. Math. 66 (1944), 299-312. MR 5, 262.

3. Y.-M. Chen, On conjugate functions, Canad. J. Math. 15 (1963), 486-494. MR 28 \#280.

4. T. M. Flett, Some theorems on odd and even functions, Proc. London Math. Soc. (3) 8 (1958), 135-148. MR 22 \#3933.

5. G. H. Hardy and J. E. Littlewood, Some more theorems concerning Fourier series and Fourier power series, Duke Math. J. 2 (1936), 354-382.

6. R. Hunt, B. Muckenhoupt and R. Wheeden, Weighted norm inequalities for the conjugate function and Hilbert transform, Trans. Amer. Math. Soc. 176 (1973), 227-251. MR 47 \# 701.

7. B. Muckenhoupt, Weighted norm inequalities for the Hardy maximal function, Trans. Amer. Math. Soc. 165 (1972), 207-226. MR 45 \#2461.

8. P. G. Rooney, On the ranges of certain fractional integrals, Canad. J. Math. 24 (1972), 11981216. MR 46 \#249.

9. A. Zygmund, Trigonometric series. Vols. I, II, Cambridge Univ. Press, New York, 1968. MR 38 \# 4882.

Department of Mathematics, University of Alberta, Edmonton, Alberta, Canada 